\title{
THE ACTIVITY OF POLYMYXINS AGAINST ESCHERICHIA COLI IN AN IN-VITRO MODEL OF THE URINARY BLADDER
}

\author{
D. GReENWOOd AND F. O'Grady \\ Department of Microbiology, University of Nottingham, City Hospital, \\ Nottingham NG5 1PH
}

THE comparative activity against Escherichia coli of polymyxin B, colistin (polymyxin E) and their sulphomethyl derivatives, sulphomyxin and colistin sulphomethate, have been examined in a previous study (Greenwood, 1975). When tested in conditions of high bacterial population density, such as may occur in infection, the activities of these polymyxins were found to differ in a way that could not be predicted on the basis of conventional MIC titrations. Polymyxin B and colistin displayed similar lytic activity against $E$. coli, but differed in the ease with which adaptive, facultatively resistant variants emerged in their presence. Sulphomyxin also possessed considerable intrinsic lytic activity, but colistin sulphomethate caused a slow, prolonged lysis of the culture consistent with recruitment of activity as the sulphomethylated derivative broke down to the more active parent compound. Adaptation to "resistance" was even easier to induce with the sulphomethyl polymyxins than with their parent compounds.

In an attempt to assess the significance of these results for therapeutic practice, we have now extended the earlier study by exposing $E$. coli to polymyxins in an in-vitro model which simulates some of the complex conditions in which bacteria and antibiotic interact in the urinary bladder.

\section{MATERIALS AND METHODS}

E. coli strain ECSA1, originally isolated from infected urine and used in the previous study of polymyxins (Greenwood, 1975) was used. Growth medium was the "complete" broth described by Greenwood and O'Grady (1973). Polymyxin B sulphate (Aerosporin) and sulphomyxin sodium (Thiosporin) were provided by Burroughs Wellcome and Co., Euston Rd, London NW1; colistin sulphate (Colomycin) and colistin sulphomethate sodium (Colomycin Injection) were supplied by Pharmax Ltd, Bourne Road, Bexley, Kent. Antibiotic solutions were prepared freshly in sterile distilled water as required.

Turbidimetric studies of static cultures were made in the twelve-channel bacterial-growthmonitoring device of Mackintosh et al. (1973). Cultures were grown from small inocula in broth and antibiotic was added at a standard point in the logarithmic growth phase ( $30 \%$ of maximum opacity).

The design of the in-vitro bladder model and its application to the study of antibiotic effects have been described elsewhere (O'Grady et al., 1973; Greenwood, 1976). In this device, a fully grown broth culture of bacteria is diluted with fresh broth at a rate of $1 \mathrm{ml}$ per min. (the normal diurnal rate of secretion of urine into the bladder) and at hourly intervals a "micturition" episode empties the "bladder", leaving a residual volume of $20 \mathrm{ml}$. Changes in the turbidity of the culture are continuously monitored photometrically.

To simulate conditions encountered in vivo, arrangements were made for the antibiotic to be instilled in gradually increasing concentrations into the "bladder" over a period of $8 \mathrm{~h}$, by use of an automatic gradient former (MixoGrad; Gilson Medical Electronics, Villiersle-Bel, France) linked to twin reservoirs of broth, one containing antibiotic at the required peak concentration, the other containing no antibiotic. Once the maximum concentration

Received 9 July 1976; revised version accepted 10 Nov. 1976.

J. MED. MICROBIOL.-VOL. 10 (1977) 
of antibiotic had been achieved, it was maintained for the reminder of the experiment, including an 8-h "sleep" period during which the inflow rate was reduced to $0.25 \mathrm{ml}$ per min. (the normal nocturnal rate of secretion of urine) and " micturition" was suspended. This "sleep" period was commenced as soon as the antibiotic concentration had reached its peak.

\section{RESULTS}

The general form of bacterial growth in the model has been described in previous communications (O'Grady et al., 1973; Greenwood and O'Grady, 1974, 1976; Greenwood, 1976): as the overnight broth culture is diluted, a fall in opacity occurs with little evidence of bacterial growth, but as the culture emerges from the lag phase bacterial growth counteracts the dilution effect until, after several hours, a "fluctuating equilibrium " state is achieved in which the same cycle of opacity changes occurs between " micturition" episodes. This form of response is illustrated in the figure (trace A).

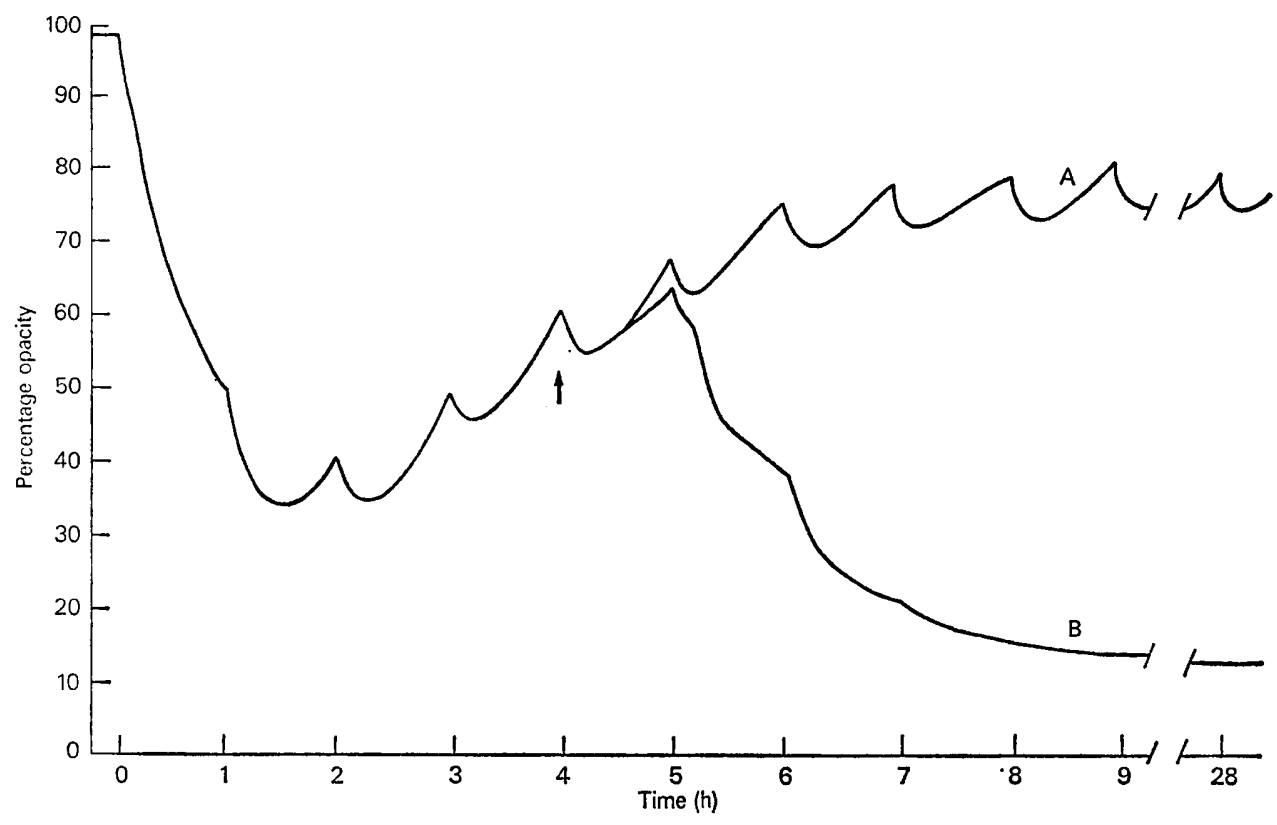

FIGURE.-Continuous opacity traces of cultures of $E$. coli in the bladder model: trace A, dilution with antibiotic-free broth throughout; trace $B$, dilution with antibiotic-free broth for the first $4 \mathrm{~h}$ after which the concentration of polymyxin in the inflow increased linearly to a maximum of $50 \mathrm{mg}$ per litre over the next $8 \mathrm{~h}$ and was subsequently maintained at that level for the remainder of the experiment. Arrow $(\uparrow)$ : exposure to antibiotic starts.

Response to polymyxin B. Preliminary experiments were performed to establish the nature of the response of cultures to a pulse of polymyxin in the conditions of the bladder model and in static culture. The susceptibility of $E$. coli to polymyxin B in the bladder model differed from that found in the static system, and in both systems some experimental variation was encountered in the polymyxin concentration needed to achieve an antibacterial effect. When a pulse of polymyxin B was added to the bacterial culture in the bladder model and the antibiotic concentration maintained by continuing dilution with antibiotic-containing broth, a concentration of between $8-16 \mathrm{mg}$ per litre was required to induce a fall in opacity. In parallel experiments in the static system, polymyxin $B$ at a concentration of $1-2 \mathrm{mg}$ per litre sufficed to induce a rapid fall in opacity. 
When polymyxin B was presented to the culture in gradually increasing concentration achieving a maximum concentration of $50 \mathrm{mg}$ per litre after $8 \mathrm{~h}$, a fall in opacity occurred as the concentration rose to an effective level (about $10 \mathrm{mg}$ per litre). If the maximum polymyxin concentration of $50 \mathrm{mg}$ per litre, once achieved, was subsequently maintained (as would be the case in a conventional therapeutic regime) suppression of growth continued for the duration of the experiment (exposure to antibiotic for a total of $24 \mathrm{~h}$; see figure, trace B); this included an 8-h (" sleep ") period during which " micturition" was suspended and the inflow rate was reduced to $0.25 \mathrm{ml}$ per min. In similar experiments in which a maximum polymyxin B concentration of $10 \mathrm{mg}$ per litre was achieved, very little effect on the normal growth pattern was observed.

Response to colistin. Colistin sulphate appeared more active than polymyxin B but gave a similar pattern of results. Colistin sulphate added to a concentration of 4-8 mg per litre and maintained at this concentration in the inflow caused a fall in opacity in the bladder model. In parallel experiments in the static system a concentration of $0.5-1.0 \mathrm{mg}$ per litre was sufficient to induce lysis of $E$. coli.

In experiments simulating the gradual excretion of colistin sulphate into the culture, a fall in opacity was observed as the concentration of colistin in the infusion rose to about $6 \mathrm{mg}$ per litre and suppression of bacterial growth was maintained for the duration of the experiment $(24 \mathrm{~h})$, even when the maximum concentration of colistin achieved was only $10 \mathrm{mg}$ per litre.

Response to sulphomethylpolymyxins. In experiments in the bladder model in which sulphomyxin and colistin sulphomethate were instilled in the culture in gradually increasing concentrations to achieve a final concentration of $50 \mathrm{mg}$ per litre in $8 \mathrm{~h}$, little effect on the normal growth pattern was observed.

\section{Discussion}

In conventional in-vitro tests, bacteria and antibiotic interact in a way that bears little relation to the therapeutic situation. For example, in the urinary bladder, antibiotic frequently has to act against a dense bacterial population that is diluted continuously by the secretion of urine (bearing a variable amount of antibiotic) and periodically discharged on micturition. Models such as the one used here seek to simulate certain important features of the clinical situation in an attempt to understand the response of bacteria to antibiotics in these dynamic circumstances.

In a previous study in a static turbidimetric system (Greenwood, 1975) exposure of dense populations of $E$. coli to polymyxins was found to select out a phenotypically resistant fraction of the population that would appear to threaten the therapeutic efficacy of these drugs. The present experiments were designed to attempt to assess whether opportunity for such variants to emerge could occur in the situation likely to be encountered in clinical practice in which a dense bacterial population is exposed to a gradually increasing polymyxin concentration.

Preliminary experiments in the bladder model to establish whether the response of the bacterial culture to a constant level of polymyxin in fluctuating growth conditions differed from that seen in static culture unexpectedly revealed a greater resistance to polymyxin in the model system than in the static system. The explanation for this phenomenon probably lies in the different metabolic state of the culture in the two systems, perhaps reflecting differences in lipid components of the cell membrane (Marr and Ingraham, 1962; Cronan, 1968). Similar increased resistance of Pseudomonas aeruginosa to polymyxin has been reported for bacteria growing in a chemostat (Melling, Robinson and Ellwood, 1974) with which the constantly diluted " bladder" culture has something in common (Mackintosh, Watson and O'Grady, 1975).

When polymyxin $\mathrm{B}$ or colistin were presented to the bacterial culture in slowly increasing concentration over a period of $8 \mathrm{~h}$, lysis of the culture occurred as the concentration reached an effective level, and no tendency for resistant variants to emerge was detected even when the final concentration of antibiotic attained only just exceeded the minimum effective level in the model system established in the preliminary experiments. 
The failure to demonstrate the emergence of phenotypic resistance in the simulated therapeutic situation probably reflects the fact that such " resistance " is due to the selection of a small pre-existing fraction of the population that tends to be suppressed by the hydrokinetic washout effect of the model-an effect that would be even more pronounced in vivo in patients having a normal residual bladder volume of about $1 \mathrm{ml}$ (Shand et al., 1968) instead of the larger residual volume $(20 \mathrm{ml})$ of the present model.

Also, increased resistance to polymyxins is probably due to a shift in lipid composition of the bacterial membrane, which, judging from the decreased susceptibility observed in the bladder model, bacteria growing in such semi-continuous culture have already undergone. Because polymyxins act directly on a preformed structure (the bacterial membrane) opportunities for greater resistance to develop do not readily arise.

The extent to which the model reflects the in-vivo situation, and hence the likely outcome of therapy, is particularly difficult to assess in the case of polymyxins. Contrasting results obtained with the bladder model and the static system highlight the important effect that environmental factors may have on the metabolic state of the bacteria and on their composition (Ellwood and Tempest, 1972). The membrane target of the polymyxins is likely to be particularly labile in this respect. Investigation of the probable efficacy of sulphomethylpolymyxins is beset by the added difficulty that these derivatives spontaneously break down to the more active parent compound, so that therapeutic activity depends on the degree and completeness of sulphomethylation, the stability of the sulphomethylated derivatives and their intrinsic activity. Antibacterial activity, therefore, is likely to be affected by variable factors in the bacteria and in the drug in a manner which is difficult to predict. Nevertheless, the failure to achieve any antibacterial effect with sulphomethylpolymyxins in the bladder model highlights the relative inactivity of these compounds in comparison with the parent antibiotics even when bacteria that appear "sensitive" in conventional tests are exposed to them for prolonged periods.

\section{SUMMARY}

The activities against a strain of Escherichia coli of polymyxin B, colistin (polymyxin E) and their sulphomethyl derivatives sulphomyxin and colistin sulphomethate have been examined in an in-vitro model of the urinary bladder under conditions similar to those that may operate in the therapeutic situation. In the dynamic conditions of the model, polymyxins exhibited a reduced activity against $E$. coli in comparison with activity against exponentially growing cultures in a static system. Nevertheless, long-term suppression of bacterial growth was achieved with levels of polymyxin B and colistin that can be attained during therapy, whereas sulphomethylpolymyxins had little effect on bacterial growth even on prolonged exposure.

\section{REFERENCES}

Cronan, J. E. JR 1968. Phospholipid alterations during growth of Escherichia coli. J. Bact., 95, 2054.

Ellwood, D. C. AND Tempest, D. W. 1972. Effect of environment on bacterial wall content and composition. In Advances in microbial physiology, vol. 7, edited by A. H. Rose and D. W. Tempest, London, p. 83.

GreENWOOD, D. 1975. The activity of polymyxins against dense populations of Escherichia coli. J. gen. Microbiol, 91, 110.

GREENWOOD, D. 1976. Use of an in vitro model of the urinary bladder in the investigation of bacterial response to antibiotics . In Chemotherapy, vol. 2, edited by J. D. Williams and A. M. Geddes, New York, p. 241.

GreENWOOD, D. AND O'GradY, F. 1973. Comparison of the responses of Escherichia coli and Proteus mirabilis to seven $\beta$-lactam antibiotics. J. infect. Dis., 128, 211.

GreENWOOD, D. AND O'Grady, F. 1974. The comparative performance of beta-lactam antibiotics against ampicillin sensitive Escherichia coli in conditions simulating those of the infected urinary bladder. Br. J. exp. Path., 55, 245. 
Greenwood, D. AND O'Grady, F. 1976. Factors influencing the response of Escherichia coli to antibiotics in conditions prevailing in the infected urinary bladder. Br.J. exp. Path., 57, 303.

Mackintosh, I. P., O’Grady, F., Greenwood, D., Watson, B. W., Crichton, T. C., PIPER, R. AND FERRER, A. 1973. A twelve channel bacterial growth monitoring system. Bio-med. Engng, 8, 514.

Mackintosh, I. P., Watson, B. W. ANd O'Grady, F. 1975. Theory of hydrokinetic clearance of bacteria from the urinary bladder. II. Effect of "bound" organisms and diuresis. Invest. Urol., 12, 473.

MARR, A. G. AND INGRAHAM, J. L. 1962. Effect of temperature on the composition of fatty acids in Escherichia coli. J. Bact., 84, 1260.

Melling, G. J., Robinson, A. AND Ellwood, D. C. 1974. Effect of growth environment in a chemostat on the sensitivity of Pseudomonas aeruginosa to polymyxin B sulphate. Proc. Soc. gen. Microbiol., 1, 61 (abstract).

O'Grady, F., Mackintosh, I. P., Greenwood, D. and Watson, B. W. 1973. Treatment of "bacterial cystitis" in fully automated mechanical models simulating conditions of bacterial growth in the urinary bladder. Br. J. exp. Path., 54, 283.

Shand, D. B., Mackenzie, J. C., CATtell, W. R. and Cato, J. 1968. Estimation of residual urine volume with ${ }^{131}$ I-hippuran. Br. J. Urol., 40, 196. 